\title{
Complementary Glycomic Analyses of Sera Derived from Colorectal Cancer Patients by MALDI-TOF-MS and Microchip Electrophoresis
}

\author{
Christa M. Snyder, William R. Alley Jr., Margit I. Campos, Martin Svoboda, John A. Goetz, \\ Jaqueline A. Vasseur, Stephen C. Jacobson, and Milos V. Novotny ${ }^{*}$ \\ Department of Chemistry, Indiana University, Bloomington, IN 47405
}

\begin{abstract}
Colorectal cancer is the fourth most prevalent cancer in the United States, yet there are no reliable non-invasive early screening methods available. Serum-based glycomic profiling has the necessary sensitivity and specificity to distinguish disease states and provide diagnostic potential for this deadly form of cancer. We applied microchip electrophoresis and MALDI-TOF-MS-based glycomic procedures to 20 control serum samples and 42 samples provided by patients diagnosed with colorectal cancer. Within the identified glycans, the position of fucose units was located to quantitate possible changes of fucosyl isomeric species associated with the pathological condition. MALDI-MS data revealed several fucosylated tri- and tetra-antennary glycans which were significantly elevated in their abundance levels in the cancer samples and distinguished the control samples from the colorectal cancer cohort in the comprehensive profiles. When compared to other cancers studied previously, some unique changes appear to be associated with colorectal cancer, being primarily associated with fucosyl isomers. Through MS and microchip electrophoresisbased glycomic methods, several potential biomarkers were identified to aid in the diagnosis and differentiation of colorectal cancer. With its unique capability to resolve isomers, microchip electrophoresis can yield complementary analytical information to MS-based profiling.
\end{abstract}

\section{Keywords}

Colorectal cancer; glycomics; mass spectrometry; microchip electrophoresis; fucosylation; Protein $\mathrm{L}$

\section{Introduction}

Blood serum is an abundant source of biochemical information pertaining to the overall state-of-health of an individual. Among its numerous major and minor proteins, glycosylated proteins abound, and their carbohydrate moieties could be particularly informative due to their important roles in biological interactions. ${ }^{1}$ Qualitative and/or quantitative deviations from "normal state" are attributes of numerous human diseases. ${ }^{2}$ In particular, aberrations in

*Corresponding Author: novotny@indiana.edu.

Supporting Information Available. Materials, sample sets, sample preparation methods, fabrication of microfluidic devices, and supporting tables and figures. This material is available free of charge via the Internet at http://pubs.acs.org. 
protein $\mathrm{N}$-glycosylation patterns $\mathrm{s}^{3-5}$ are observed due to different cancer conditions and their clinical stages. ${ }^{6-7}$ These changes have been emphasized by several research groups which have demonstrated significant changes to the healthy serum glycomic profiles for a number of cancers. ${ }^{8-13}$ Although the so-called true biomarkers will most assuredly be cell-surface glycoproteins originating from malignant cells which are shed into the blood stream and feature unique glycosylation patterns, changes to the carbohydrate patterns of many of the more highly abundant acute-phase proteins that are synthesized by the liver and secreted into the circulatory system, along with the various immunoglobulins, may present potentially more analytically reachable and effective ways to monitor disease progression or the effectiveness of therapy programs.

In biomarker discovery, identifying the specificity of serum glycomarkers for a particular disease condition is important. To address this issue, we have examined the altered glycosylation patterns of several different cancers by mass spectrometry (MS) and microchip electrophoresis -based glycomic methods, with some glycans appearing to be at least somewhat unique to a particular cancer. The specificity of some indicators, at this early stage, seems to be further accentuated by studying the positional isomers of fucosylated glycans ${ }^{10,14}$ and the different possible linkage isomers of sialic acids. ${ }^{14-16}$

In this study, we examined the serum glycomic profiles from colorectal cancer samples. Colorectal cancer is the fourth most prevalent cancer in the United States, behind only lung, breast, and prostate cancers, ${ }^{17,18}$ and is the fourth most deadly cancer in the world, behind lung, liver, and stomach cancers. ${ }^{19}$ Over 132,000 new cases are diagnosed each year, and 50,000 annual deaths are caused by this disease. ${ }^{17,18}$ Most often, colorectal cancer is detected with invasive procedures such as colonoscopy or double-contrast barium enemas. Less invasive techniques, such as immunochemical fecal occult blood tests (iFOBTs), exist and give an improvement over previous methods, but suffer from lower sensitivity and specificity when compared to colonoscopies. ${ }^{20}$ Therefore, patients and clinicians alike are keenly interested in the development of non-invasive diagnostic tests that maintain high sensitivity and specificity. Due to the ease of collection, a procedure based on blood is highly desirable. Serum-based methods, such as the detection of methylated SEPT9 DNA, ${ }^{21}$ can detect up to $68 \%$ of colorectal cancer cases, but this test is crippled by a $20 \%$ false positive rate and low sensitivity for early-stage cancers. ${ }^{22}$ Another plasma-based assay uses carcinoembryonicantigen (CEA), a glycoprotein containing $60 \%$ carbohydrate by mass, ${ }^{23}$ and is currently the most promising biomarker to detect colorectal cancer from serum samples. ${ }^{24,25}$ However, like SEPT9, CEA does not have enough reliability or sensitivity to detect very early stages of the disease. ${ }^{4,26}$

Given its high rates of incidence and mortality, the fact that only a few glycoconjugate-based studies have been conducted for colorectal cancer is somewhat surprising. In one of these studies, the N-linked glycans of colorectal cancer tissues were studied and indicated decreased levels of bisecting glycans, and core-fucosylated high mannose-type glycans were also observed only in the tumor tissues. ${ }^{27}$ In a second study, O-linked glycopeptides featuring aberrant glycosylation patterns originating from MUC1 and MUC2 identified several autoantibodies that were capable of diagnosing colon cancer with a specificity of $92 \%$ and a sensitivity of $79 \% .{ }^{28}$ Recently, MALDI-TOF-MS ${ }^{29,30}$ and DNA sequencer- 
assisted fluorophore-assisted capillary electrophoresis (DSA-FACE) ${ }^{23}$ were used to analyze $\mathrm{N}$-glycosylation from colorectal cancer patients and control patients. These experiments showed good differentiation between sample sets but omitted isomer identification and peak information. Similarly to other cancers, fucosylation seems to play an important role in colorectal cancer, with elevated levels of fucosylated haptoglobin being associated with distant metastases and a poorer overall prognosis. ${ }^{31}$

One of the primary objectives of our laboratories has been the analysis of different pathological conditions to identify possible glycomarkers, both at the level of glycoproteins and released glycans, with a primary focus on cancer. In addition to tentatively documenting possible molecules to indicate the presence of a disease, we are also interested in the ability of serum-based glycomarkers to diagnose a specific condition. We have demonstrated alterations to the control serum-based profiles for breast, ${ }^{32}$ prostate, ${ }^{33}$ esophageal, ${ }^{34-35}$ ovarian, ${ }^{7,} 10$ and lung cancers, ${ }^{14}$ in addition to several highly fucosylated glycans present in some pre-malignant pancreatic cyst fluids. ${ }^{36}$ In some of these cases, certain glycomic alterations may be specific to a given cancer, and this specificity may also be improved through an examination of fucosyl ${ }^{10,14,37}$ and sialic-acid linkage isomers. ${ }^{15}$ In this study, we extend our glycomic investigations to colorectal cancer. We have subjected control and colorectal cancer serum samples to comprehensive glycomic profiling analyses by both microchip electrophoresis and MALDI-TOF-MS. Microchip electrophoresis resolves structural isomers and is highly reproducible, whereas MALDI-TOF-MS provides more definitive structural information, e.g., the position of fucose residues and possible alterations in these positions associated with disease progression.

\section{Experimental}

The materials, samples sets, and sample preparation steps are described in the Supporting Information. The sample preparation steps include PNGase F digestion and solid-phase extraction, reduction of N-glycans for MALDI-TOF-MS, digestion of N-glycans with exoglycosidases, and spin-column permethylation of N-glycans.

\section{MALDI-TOF-MS Analysis}

The N-linked glycans for each analysis were permethylated according to our previously published protocol. ${ }^{38}$ Samples were reconstituted in a 5- $\mu \mathrm{L}$ aliquot of a 50\%/50\% water/ methanol solution. A $0.5-\mu \mathrm{L}$ aliquot of each sample was spotted on a MALDI plate and allowed to dry. Each sample was spotted in triplicate. Subsequently, a $0.5-\mu \mathrm{L}$ aliquot of a 10 $\mathrm{mg} / \mathrm{ml}$ 2,5-DHB/1 $\mathrm{mM}$ sodium acetate solution was added to each spot and dried under vacuum. The samples were analyzed by an Applied Biosystems 4800 MALDI-tandem TOFMS instrument (Foster City, California) and measured automatically in the instrument's batch mode. The instrument was operated in its positive-ion mode, monitoring the $\mathrm{m} / \mathrm{z}$ range from 1500 to 5000 . A total of 1,000 laser shots were acquired for each sample spot.

\section{MALDI-TOF-MS Data Processing and Statistical Analysis}

Spectra were base-line corrected, a noise filter was applied, and the data converted to text files. The data were normalized by expressing the intensity of each glycan ion as a 
percentage of the total intensity for all glycans included in this study. (For a list of these glycans, see Table S1 in the Supporting Information.) Following normalization, the three spectra for each sample were averaged and then subjected to a series of statistical tests, including one-way analysis-of-variance (ANOVA), which was performed with Microsoft Excel 2013. Glycomic data resulting in statistically-significant $p$-values (less than 0.05 in this study) were further processed by a receiver-operator characteristics (ROC) test in Origin 9.0 (OriginLab Corp., Northampton, MA), which produced an area-under-the-curve (AUC) value that ranged from 0.0 to 1.0. To determine the significance of the values, a slight modification to the arbitrary guidelines proposed by Swets ${ }^{39}$ was used. According to these recommendations, if the AUC value was greater than 0.9 or less than 0.1, the tests were considered highly accurate, whereas values between $0.8-0.9$ or $0.1-0.2$ were deemed as accurate. When the AUC value was between $0.7-0.8$ or between $0.2-0.3$, the test was moderately accurate. An uninformative test resulted in an AUC value that was between 0.3 and 0.7 .

\section{Methylamidation and Fluorescent Labeling of N-Glycans for Microchip Electrophoresis}

The sample set to be analyzed by microchip electrophoresis was methylamidated, as reported previously, ${ }^{40}$ for a direct comparison to MALDI-TOF-MS data and then labeled with $\mathrm{APTS}^{41}$ by the established procedures ${ }^{42}$ to impart a negative charge for electrophoresis and a fluorescent tag for detection. See the Supporting Information for additional reaction details.

\section{Fabrication of Microfluidic Devices}

We used standard photolithography, wet chemical etching, and cover plate bonding to fabricate the microfluidic devices. ${ }^{42}$ Microchannels were coated with linear polyacrylamide to suppress the electroosmotic flow. See the Supporting Information for further details on the fabrication process.

\section{Microchip Electrophoresis}

The all-glass microfluidic device had a serpentine channel with a 22-cm separation length and asymmetrically tapered, $180^{\circ}$ turns (Figure S2 in the Supporting Information). ${ }^{43}$ Potentials were applied to the sample, buffer, and waste reservoirs with a fast-slewing highvoltage power supply $(0-10 \mathrm{kV})$ and to the analysis reservoir with a commercial highvoltage power supply (0-30 kV; CZE 1000R, Spellman High Voltage Electronics Corp., Hauppauge, NY). The high voltage outputs were controlled through an analog output board (PCI-6713, National Instruments Corp.) by a program written in LabVIEW 8.2 (National Instruments Corp., Austin, TX). Samples were introduced into the analysis channel by standard and modified pinched injections. ${ }^{42,} 44$

Separations were monitored on an inverted optical microscope (TE-2000U, Nikon, Inc., Tokyo, Japan) configured for epifluorescence and equipped with a 20× objective and HQ FITC filter cube (Chroma Technology Corp., Bellows Falls, VT). The 488-nm line of an argon ion laser (Melles Griot, Inc., Albuquerque, NM) was attenuated to $1.4 \mathrm{~mW}$ with neutral density filters and focused to a spot in the analysis channel 22-cm downstream from the cross intersection. The fluorescence signal was spatially filtered with a $400-\mu \mathrm{m}$ pinhole, 
detected with a photomultiplier tube (H5783-01, Hamamatsu Corp., Shizuoka, Japan), amplified by a low-noise current preamplifier (SR570, Stanford Research Systems, Inc., Sunnyvale, CA), and recorded at $100 \mathrm{~Hz}$ with a multifunction data acquisition board (PCI-6032E, National Instruments Corp.) and the LabVIEW program.

\section{Microchip Electrophoresis Data Analysis}

Peaks were manually fitted to a Gaussian function in OriginPro 2015 for all electropherograms. Peak areas from three replicate electropherograms of each sample were averaged, and each peak was normalized to the total area sum of all peaks in the averaged electropherogram. Before performing principal component analyses (PCA), the data were found to be normally distributed. Supervised PCA was carried out with prior knowledge of the sample groups with MarkerView 1.2.1 (Applied Biosystems). $p$-Values were calculated through single-variable pairwise ANOVA parametric analysis with SPSS 20 (IBM Corp., North Castle, NY). OriginPro 2015 was again used to perform ROC tests and generate AUC values to determine the diagnostic potential of each peak as a test.

\section{Results and Discussion \\ Comprehensive MALDI-TOF-MS Glycomic Profiles}

MALDI-TOF-MS was performed on control $(\mathrm{N}=20)$ and colorectal serum samples from patients diagnosed with colorectal cancer after a first treatment cycle ("C1 samples", $\mathrm{N}=$ 26) and a third treatment cycle ("C 3 samples", $\mathrm{N}=16$ ). We were unable to obtain an appropriate cohort of inflamed samples that would have been reflective of the ages and genders of the control and cancer cohorts. These patients were men and women who were diagnosed with either colon or rectal cancer and ranged in age from 25-77 years old with a variety of prior smoking habits and previous cancer treatment methods, e.g., radiation therapy, chemotherapy, and surgery (selected meta-data can be found in Table S2). MALDITOF-MS glycomic profiles of serum resulted in the detection of approximately 45 unique ion masses that correspond to previously characterized ${ }^{14,45-46} \mathrm{~N}$-glycan structures. A representative profile for a colorectal cancer serum sample is shown in Figure 1. For these types of spectra, we apply relative quantitation methods to probe their possible differences between the different states-of-health. The ion intensities for all $\mathrm{N}$-glycan were summed and individual ion intensities were expressed as a percentage of the total sum. Pair-wise comparisons of relative intensities were compared to identify the extent of differentiation between sample groups. In this study of the control, C1, and C3 sample groups, ANOVA testing indicated significant differences between the control and $\mathrm{C} 1$ groups for 11 glycans and between control and C3 samples for 19 glycans, as based on a p-value of less than 0.05.

This subset was then further processed by an ROC test, and all $p$-values and AUC values can be found in Table S1 in the Supporting Information. With a cut-off of 0.7 (or 0.3 for a negative test) for the AUC values, 4 glycans could differentiate $\mathrm{C} 1$ cancer samples from the control samples, 16 glycans could differentiate $\mathrm{C} 3$ cancer samples from the control samples, and 7 glycans could differentiate the combination of $\mathrm{C} 1$ and $\mathrm{C} 3$ from the control samples. From this subset of oligosaccharides, three were fucosylated tri- or tetra-antennary glycans. These glycans are typically found in the higher mass region of the spectrum and are shown 
as an inset for Figure 1. These particular types of glycans were also primarily responsible for distinguishing both ovarian ${ }^{10}$ and lung ${ }^{14}$ cancer samples from control samples in our previous studies and have been tentatively identified as predictive biomarkers for prostate cancer. ${ }^{13}$ The normalized intensity data for selected glycans are shown as the notched box plots in Figure 2.

Such similarities between different cancers are beginning to indicate the need for more detailed investigations. Studies of liver diseases ${ }^{47}$ and pancreatic cancer ${ }^{48}$ have demonstrated that haptoglobin bears enhanced levels of more highly branched fucosylated glycans, which are seemingly reflected in our profiles. However, these types of glycans were attached to different sites in each disease, and the glycopeptides may thus indicate an inherent level of specificity for a particular disease. Therefore, our future studies need to identify the protein(s) featuring the tri- and tetra-antennary glycans and characterize them in more detail to improve the specificity of serum glycomarkers.

The most striking changes among the different states-of-health were observed for a fucosylated tetra-antennary glycan bearing four sialic acids (recorded at an $\mathrm{m} / \mathrm{z}$ value of 4603), and singly- and doubly-fucosylated tri-antennary/tri-sialylated glycans (observed at $\mathrm{m} / \mathrm{z}$ values of 3793 and 3966 , respectively). All three glycans were found in relative abundances greater than $0.20 \%$ in the control samples, but had abundances up to two times larger for cancer samples. The AUC values for the glycans at $m / 2$ values of 3793 and 3966 were calculated to 0.75 or greater. Interestingly, the non-fucosylated tetra-antennary glycans appeared to have consistent relative abundances, regardless of the state-of-health in this study, which was also observed in our previous lung cancer study. ${ }^{14}$ These glycan structures, however, were recorded with elevated abundances in our earlier ovarian cancer study. ${ }^{10}$

Based on our previous studies, ${ }^{10,49}$ three remaining glycans have all been tentatively associated with the various immunoglobulins. In our previous cancer glycomic studies, we, along with others, have observed a general trend of decreased abundances of galactosylated glycans and elevated levels of those carbohydrates lacking this particular monosaccharide. ${ }^{10,14,45}$ In this study, the abundance levels of the non-galactosylated glycans remained constant between the two samples sets, whereas suppressed levels of some galactosylated glycans were observed in the cancer cohort. This change was most prominent for a core-fucosylated, biantennary/digalactosylated glycan $(\mathrm{m} / \mathrm{z}$ value of 2260$)$, with the data associated with this glycan producing an AUC value of 0.136 for control vs $C 3$. The other two Ig-associated glycans both possessed a "bisecting" GlcNAc unit and were elevated in their abundances in the cancer samples, resulting in AUC scores greater than 0.7. The relative intensity for the glycan associated with an $\mathrm{m} / \mathrm{z}$ value of 1922 (see Table S1 in the Supporting Information for its proposed structure) was nearly doubled in the $\mathrm{C} 1$ sample set and almost tripled in the $\mathrm{C} 3$ sample set. This glycan was not observed to be elevated in its abundance level in the total profiles for ovarian or lung cancer. The other bisecting glycan, present at an $\mathrm{m} / \mathrm{z}$ value of 2662 , was elevated in lung cancer, ${ }^{14}$ but not ovarian cancer. ${ }^{10}$ The elevated levels of these two IgG-associated glycans in tandem may be a unique feature to colorectal cancer. 


\section{MALDI-TOF-MS Analysis of Fucosyl Isomers}

One of our interests has been to uncover more information contained within the serum glycome beyond that which is provided by the comprehensive profiles. To do this, we have begun a more rigorous study of differences of the sialic-acid linkages ${ }^{10}$ and the changes of their ratios induced by a disease. ${ }^{14-15} \mathrm{We}$ are also examining the differences in the isomeric species of fucosylated glycans, ${ }^{10,14}$ where the fucosyl substitutions can be located on the core- or an outer-arm. Fucosyl isomers can be distinguished by MS through an exoglycosidase treatment with a non-specific sialidase and a $\beta$-galactosidase, whose action is inhibited by outer-arm fucoses. ${ }^{50}$ Following this digestion, the mass difference between core and outer-arm fucosylated glycans from a parent carbohydrate will be that of an attached galactose. This treatment simplifies the spectrum to about 15 structures which can be reliably quantitated.

We have demonstrated previously the value of performing an analysis of fucosyl isomers. Although elevated levels of the same fucosylated glycans (for example, that with an $\mathrm{m} / \mathrm{z}$ value of 3792) have been observed in lung, ${ }^{14}$ ovarian,,${ }^{10}$ and liver ${ }^{51}$ cancers in the comprehensive profiles, an analysis of the location of the fucose unit has been able to distinguish lung and ovarian cancers, where outer-arm fucosylation was the favored position, ${ }^{10,14}$ from liver diseases where core fucosylation is elevated (unpublished results). Different abundance ratios for fucosyl isomers were also observed in this study, some of which may be unique to colorectal cancer. Interestingly, the levels of core fucosylation in colorectal cancer seem to be elevated for tri-antennary glycans, a trend which is further accentuated for the tetra-antennary class (see the notched box plots in Figure S1 in the Supporting Information), where this type of fucosylation was increased nearly three times in the cancer samples. Elevated levels of outer-arm fucosylation associated with the tetraantennary glycans were also observed in the cancer cohort, again by a factor of three, but such a change was not observed for the tri-antennary structures. For the different cancers, that we have studied to date with this approach, ${ }^{10,14}$ this combination of increased amounts of both core and outer-arm fucosylation appears to be unique to colorectal cancer. The potential importance of increased levels of core fucosylation were also indicated by the nearly doubled abundance levels of the tri-antennary glycans possessing both core and outerarm substitutions in the pathological samples. Finally, a doubly-outer-arm tetra-antennary product was not observed in many of the control samples, but was detected in the majority of cancer samples. This alteration is also consistent with those from our ovarian and lung cancer samples. ${ }^{10,14}$

\section{Glycomic Profiles from Microchip Electrophoresis}

In a complementary set of experiments, microchip electrophoresis was performed on a second aliquot of the same serum samples analyzed by MALDI-TOF-MS. Samples were methylamidated prior to analysis, and these $\mathrm{N}$-glycans migrated in a window from 80 to 135 s, as shown in Figure 3. Separation efficiencies were up to 700000 theoretical plates, which is comparable, if not better per unit length, than traditional capillary separations. ${ }^{42}$ This efficiency gives good resolution between both low- and high-abundance analytes. Triplicates of electropherograms boasted migration time RSD values of less than $0.034 \%$ across the 55 s migration window, as well as peak area RSD variations of less than $5.9 \%$, both of which 
highlight the excellent reproducibility of this method. A schematic of the device used can be found as Figure S2 in the Supporting Information.

A series of statistical tests was performed with the peak areas from the electropherograms. Methylamidated $\mathrm{N}$-glycans from control, $\mathrm{C} 1$, and $\mathrm{C} 3$ sample groups were compared through PCA, as shown in Figure 4a-b. There is significant differentiation among the three groups despite the differences in age, gender, and diagnosis for all samples (Table S2). Moreover, the clustering within each sample group indicates that there are commonalities within each group and discernible differences among groups, most likely due to the presence of cancer in different stages. The removal of two of these variables in $\mathrm{C} 1$ and $\mathrm{C} 3$ samples by sex-andage-matching results in greater separation among the groups, as shown in Figure 4b. This increase in differentiation is in good agreement with previous results ${ }^{6-7}$ which showed enhanced differentiation in more tightly controlled sample groups. Additionally, enhanced differentiation is seen in several other age and gender groupings (see Figure S3 in the Supporting Information).

Pairwise comparison of normalized peak areas for control and C1, control and C3, and C1 and $\mathrm{C} 3$ were carried out to identify statistical differences among the sample populations. Figure 5 shows peaks that had $p$-values $<0.1$ for at least one comparison. Of the 24 total peaks with $p$-values $<0.1$, three of them were for control vs $\mathrm{C} 1$. Comparison of control vs $\mathrm{C} 3$ and $\mathrm{C} 1$ to $\mathrm{C} 3$ resulted in 14 and 7 significant peaks, respectively. These results are in good agreement with the relative separation between sample sets in Figure 4. The least amount of overlap was seen between the $\mathrm{C} 3$ and control samples, and the most overlap was between the control and $\mathrm{C} 1$ samples. Many of the patients' conditions worsened after continued treatment, which is reflected in the high number of significant values for control vs $\mathrm{C} 3$ samples compared to control vs $\mathrm{C} 1$. The high number of significant differences between cancer samples is indicative of the potential of this technique to separate different cancer stages from one another.

ROC plots were generated for peaks with $p$-values $<0.1$. Of the significant peaks in the comparison of control and C3 samples, peaks 18 and 20 resulted in the largest AUC values, 0.91 and 1.0, respectively, shown in Figure S4 in the Supporting Information. From the remaining 12 peaks with $p$-values $<0.1$, seven of them had AUC values $>0.7$. The comparison of control to $\mathrm{C} 1$ samples resulted in only one peak with an AUC value $>0.7$ (peak 18), but three peaks were found to have AUC values $>0.7$ for the comparison of $\mathrm{C} 1$ to C3 samples (peaks 18, 20, and 34).

\section{Comparison of MALDI-MS to Microchip Electrophoresis Results}

We previously identified and assigned specific $\mathrm{N}$-glycan compositions to peaks in electropherograms $\mathrm{s}^{52}$ and used these structural assignments to compare changes in peak areas found through electrophoresis to changes in intensities for specific masses from MALDITOF-MS analysis. Glycans detected by microchip electrophoresis that were statistically different between control and pathological samples were compared to glycans identified as significant through MALDI-TOF-MS analysis to determine the overlap of these two techniques. From these comparisons, three peaks for control vs $\mathrm{C} 1$, five peaks for control vs 
$\mathrm{C} 3$, and two peaks for control vs the combination of C1 and C3 (denoted as "cancer") were found to be significant $(p<0.1)$ in both datasets, and these results are summarized in Table 1. Seen as notable is the large, triantennary, fucosylated structure (microchip electrophoresis peak 43, $\mathrm{m} / \mathrm{z}$ 3793) that is significant for both control vs $\mathrm{C} 1$ and control vs $\mathrm{C} 3$. As previously mentioned, highly branched, heavily sialylated glycans have been indicated in cancer progression, and this trend was seen in both microchip electrophoresis and MALDI analysis methods.

Although in good agreement for several significant $\mathrm{N}$-glycan structures, many differences in these datasets arise from the inherent differences in the techniques. Unlike mass spectrometric methods, ${ }^{16}$ microchip electrophoresis has the ability to resolve structural isomers of glycans without further derivatization, which results in multiple peaks in electropherograms corresponding to one $\mathrm{m} / \mathrm{z}$ peak in a mass spectrum. The relative ratios of specific isomers may prove important for differentiating between disease states, but their combination into one $\mathrm{m} / \mathrm{z}$ value is no longer statistically relevant. Several large, tetraantennary glycans are now routinely identified via mass spectrometry, but their separation by microchip electrophoresis has not been optimized. In this way, the two techniques serve as complementary methods to obtain a full spectrum of information from a sample set.

\section{Conclusions}

Serum glycomic profiling potentially offers a non-invasive technique that can be used to identify diagnostic biomarkers for different types of cancer. We have used both microchip electrophoresis and MALDI-TOF-MS of serum N-glycans to characterize and identify different biomarker candidates to differentiate between different stages of colorectal cancer and different cancer types. These glycans are characterized by very low $p$-values for comparisons of peak intensities and areas between disease states, as well as high AUC values from ROC tests. Such high AUC values for the comparison of control to early stage cancer samples indicate potential clinical utility.

In this study, we have added to the growing library of glycomic information pertaining to cancer. Here, we further emphasize the role of fucosylation and its emerging role to identify a particular cancer, especially if the levels of both fucosylated and non-fucosylated glycans are compared for different cancers. As demonstrated here, the lack of change for a given set of glycans may hold diagnostic information and when taken together, the potential glycobiomarker classes seem to have sensitivities and specificities, two characteristics that are currently too low or altogether missing from other early diagnostic tests. Fucosyl isomer analysis further indicated the presence of colorectal cancer-specific biomarkers in the serum glycome. The use of several different cancer-specific biomarkers obtained from one sample can be used to unambiguously identify both disease and state at very high rates of sensitivity and specificity. With the inherent complexity of glycosylation, coupled with the need for indepth glycomic analyses, multimethodological and complementary analytical workflows are needed to more accurately diagnose specific cancers in their earlier stages. 


\section{Supplementary Material}

Refer to Web version on PubMed Central for supplementary material.

\section{Acknowledgments}

This work was supported by Grant No. U01 CA128535 from the National Cancer Institute, and Grant Nos. R01 GM24349 and R01 GM106084 from the National Institute of General Medical Sciences, U.S. Department of Health and Human Services. The authors also thank the Hoosier Oncology Group for providing the samples for this study and the support from the Walther Cancer Institute Foundation.

\section{Abbreviations}

$\begin{array}{ll}\text { MALDI-TOF-MS } & \begin{array}{l}\text { matrix-assisted laser desorption/ionization time-of-flight } \\ \text { mass spectrometry }\end{array} \\ \text { ANOVA } & \text { analysis-of-variance } \\ \text { ROC } & \text { receiver-operator characteristics } \\ \text { AUC } & \text { area-under-the-curve } \\ \text { PNGase F } & \text { Peptide-N-Glycosidase F } \\ \text { 2,5-DHB } & \text { 2,5-dihydroxybenzoic acid } \\ \text { SDS } & \text { sodium dodecylsulfate } \\ \text { ACN } & \text { acetonitrile } \\ \text { DMF } & \text { dimethylformamide } \\ \text { TFA } & \text { trifluoroacetic acid } \\ \text { LC } & \text { liquid chromatography } \\ \text { IgG } & \text { immunoglobulin G } \\ \end{array}$

\section{References}

1. Hart GW, Copeland RJ. Cell. 2010; 143(5):672-676. [PubMed: 21111227]

2. Varki, A. C., R.D.; Eski, J.D.; Freeze, H.H.; Stanley, P.; Bertozzi, C.R.; Hart, G.W.; Etzler, M.E., 2nd ed.; Cold Spring Harbor Press: NY, 2009.

3. Kobata A, Amano J. Immunol Cell Biol. 2005; 83(4):429-439. [PubMed: 16033539]

4. Holst S, Stavenhagen K, Balog CIA, Koeleman CAM, McDonnell LM, Mayboroda OA, Verhoeven A, Mesker WE, Tollenaar R, Deelder AM, Wuhrer M. Mol Cell Proteomics. 2013; 12(11):30813093. [PubMed: 23878401]

5. Guo HB, Nagy T, Pierce M. J Biol Chem. 2014; 289(45):31534-31549. [PubMed: 25274627]

6. Goldman R, Ressom HW, Varghese RS, Goldman L, Bascug G, Loffredo CA, Abdel-Hamid M, Gouda I, Ezzat S, Kyselova Z, Mechref Y, Novotny MV. Clin Cancer Res. 2009; 15(5):1808-1813. [PubMed: 19223512]

7. Mitra I, Alley WR, Goetz JA, Vasseur JA, Novotny MV, Jacobson SC. J Proteome Res. 2013; 12(10):4490-4496. [PubMed: 23984816] 
8. Arnold JN, Saldova R, Galligan MC, Murphy TB, Mimura-Kimura Y, Telford JE, Godwin AK, Rudd PM. J Proteome Res. 2011; 10(4):1755-1764. [PubMed: 21214223]

9. Abbott KL, Lim JM, Wells L, Benigno BB, McDonald JF, Pierce M. Proteomics. 2010; 10(3):470_ 481. [PubMed: 19953551]

10. Alley WR, Vasseur JA, Goetz JA, Syoboda M, Mann BF, Matei DE, Menning N, Hussein A, Mechref Y, Novotny MV. J Proteome Res. 2012; 11(4):2282-2300. [PubMed: 22304416]

11. Kirmiz C, Li BS, An HJ, Clowers BH, Chew HK, Lam KS, Ferrige A, Alecio R, Borowsky AD, Sulaimon S, Lebrilla CB, Miyamoto S. Mol Cell Proteomics. 2007; 6(1):43-55. [PubMed: 16847285]

12. Biskup K, Braicu EI, Sehouli J, Fotopoulou C, Tauber R, Berger M, Blanchard V. J Proteome Res. 2013; 12(9):4056-4063. [PubMed: 23889230]

13. Ishibashi Y, Tobisawa Y, Hatakeyama S, Ohashi T, Tanaka M, Narita S, Koie T, Habuchi T, Nishimura SI, Ohyama C, Yoneyama T. Prostate. 2014; 74(15):1521-1529. [PubMed: 25154914]

14. Vasseur JA, Goetz JA, Alley WR, Novotny MV. Glycobiology. 2012; 22(12):1684-1708. [PubMed: 22781126]

15. Novotny MV, Alley WR, Mann BF. Glycoconjugate J. 2013; 30(2):89-117.

16. Alley WR, Novotny MV. J Proteome Res. 2010; 9(6):3062-3072. [PubMed: 20345175]

17. Jemal A, Siegel R, Ward E, Hao YP, Xu JQ, Murray T, Thun MJ. Ca-Cancer J Clin. 2008; 58(2): 71-96. [PubMed: 18287387]

18. Siegel RL, Miller KD, Jemal A. CA Cancer J Clin. 2015; 65(1):5-29. [PubMed: 25559415]

19. Brody H. Nature. 2015; 521(7551):S1. [PubMed: 25970450]

20. Levi Z, Rozen P, Hazazi R, Vilkin A, Waked A, Maoz E, Birkenfeld S, Leshno M, Niv Y. Ann Intern Med. 2007; 146(4):244-255. [PubMed: 17310048]

21. Potter NT, Hurban P, White MN, Whitlock KD, Lofton-Day CE, Tetzner R, Koenig T, Quigley NB, Weiss G. Clin Chem. 2014; 60(9):1183-1191. [PubMed: 24938752]

22. Willyard C. Nature. 2015; 521(7551):S4-S5. [PubMed: 25970456]

23. Zhao YP, Ruan CP, Wang H, Hu ZQ, Fang M, Gu X, Ji J, Zhao JY, Gao CF. Cancer. 2012; 118(3): 639-650. [PubMed: 21853445]

24. Vercoutter-Edouart AS, Slomianny MC, Dekeyzer-Beseme O, Haeuw JF, Michalski JC. Proteomics. 2008; 8(16):3236-3256. [PubMed: 18651673]

25. Khales SA, Abbaszadegan MR, Abdollahi A, Raeisossadati R, Tousi MF, Forghanifard MM. J Cancer Res Clin Oncol. 2015; 141(2):229-235. [PubMed: 25156818]

26. Kim HJ, Yu MH, Kim H, Byun J, Lee C. BMB Rep. 2008; 41(10):685-692. [PubMed: 18959813]

27. Balog CIA, Stavenhagen K, Fung WLJ, Koeleman CA, McDonnell LA, Verhoeven A, Mesker WE, Tollenaar R, Deelder AM, Wuhrer M. Mol Cell Proteomics. 2012; 11(9):571-585. [PubMed: 22573871]

28. Pedersen JW, Blixt O, Bennett EP, Tarp MA, Dar I, Mandel U, Poulsen SS, Pedersen AE, Rasmussen S, Jess P, Clausen H, Wandall HH. Int J Cancer. 2011; 128(8):1860-1871. [PubMed: 21344374]

29. Holst S, Wuhrer M, Rombouts Y. Advances in cancer research. 2015; 126:203-56. [PubMed: 25727149]

30. Coghlin C, Murray GI. Proteom Clin Appl. 2015; 9(1-2):64-71.

31. Takeda Y, Shinzaki S, Okudo K, Moriwaki K, Murata K, Miyoshi E. Cancer. 2012; 118(12):30363043. [PubMed: 22006099]

32. Kyselova Z, Mechref Y, Kang P, Goetz JA, Dobrolecki LE, Sledge GW, Schnaper L, Hickey RJ, Malkas LH, Novotny MV. Clin Chem. 2008; 54(7):1166-1175. [PubMed: 18487288]

33. Kyselova Z, Mechref Y, Al Bataineh MM, Dobrolecki LE, Hickey RJ, Vinson J, Sweeney CJ, Novotny MV. J Proteome Res. 2007; 6(5):1822-1832. [PubMed: 17432893]

34. Mechref Y, Hussein A, Bekesova S, Pungpapong V, Zhang M, Dobrolecki LE, Hickey RJ, Hammond ZT, Novotny MV. J Proteome Res. 2009; 8(6):2656-2666. [PubMed: 19441788]

35. Mitra I, Zhuang ZX, Zhang YN, Yu CY, Hammoud ZT, Tang HX, Mechref Y, Jacobson SC. Anal Chem. 2012; 84(8):3621-3627. [PubMed: 22397697] 
36. Mann BF, Goetz JA, House MG, Schmidt CM, Novotny MV. Mol Cell Proteomics. 2012; 11(7)

37. Singh S, Pal K, Yadav J, Tang H, Partyka K, Kletter D, Hsueh P, Ensink E, Birendra KC, Hostetter G, Xu HE, Bern M, Smith DF, Mehta AS, Brand R, Melcher K, Haab BB. J Proteome Res. 2015; 14(6):2594-2605. [PubMed: 25938165]

38. Alley WR, Madera M, Mechref Y, Novotny MV. Anal Chem. 2010; 82(12):5095-5106. [PubMed: 20491449]

39. Swets JA. Science. 1988; 240(4857):1285-1293. [PubMed: 3287615]

40. Liu X, Qiu HY, Lee RK, Chen WX, Li JJ. Anal Chem. 2010; 82(19):8300-8306. [PubMed: 20831242]

41. Evangelista RA, Chen FTA, Guttman A. J Chromatogr A. 1996; 745(1-2):273-280.

42. Zhuang Z, Starkey JA, Mechref Y, Novotny MV, Jacobson SC. Anal Chem. 2007; 79(18):71707175. [PubMed: 17685584]

43. Ramsey JD, Jacobson SC, Culbertson CT, Ramsey JM. Anal Chem. 2003; 75(15):3758-3764. [PubMed: 14572041]

44. Jacobson SC, Hergenroder R, Koutny LB, Warmack RJ, Ramsey JM. Anal Chem. 1994; 66(7): 1107-1113.

45. Saldova R, Royle L, Radcliffe CM, Hamid UMA, Evans R, Arnold JN, Banks RE, Hutson R, Harvey DJ, Antrobus R, Petrescu SM, Dwek RA, Rudd PM. Glycobiology. 2007; 17(12):13441356. [PubMed: 17884841]

46. Hecht ES, Scholl EH, Walker SH, Taylor AD, Cliby WA, Motsinger-Reif AA, Muddiman DC. J Proteome Res. 2015; 14(10):4394-4401. [PubMed: 26347193]

47. Pompach P, Brnakova Z, Sanda M, Wu J, Edwards N, Goldman R. Mol Cell Proteomics. 2013; 12(5):1281-1293. [PubMed: 23389049]

48. Nakano M, Nakagawa T, Ito T, Kitada T, Hijioka T, Kasahara A, Tajiri M, Wada Y, Taniguchi N, Miyoshi E. Int J Cancer. 2008; 122(10):2301-2309. [PubMed: 18214858]

49. Svoboda M, Mann BF, Goetz JA, Novotny MV. Anal Chem. 2012; 84(7):3269-3277. [PubMed: 22360417]

50. Lucka L, Fernando M, Grunow D, Kannicht C, Horst AK, Nollau P, Wagener C. Glycobiology. 2005; 15(1):87-100. [PubMed: 15317738]

51. Kang P, Madera M, Alley WR, Goldman R, Mechref Y, Novotny MV. Int J Mass Spectrom. 2011; 305(2-3):185-198. [PubMed: 23788846]

52. Mitra I, Snyder CM, Zhou X, Campos MI, Alley JWR, Novotny MV, Jacobson SC. Anal Chem. 2016 


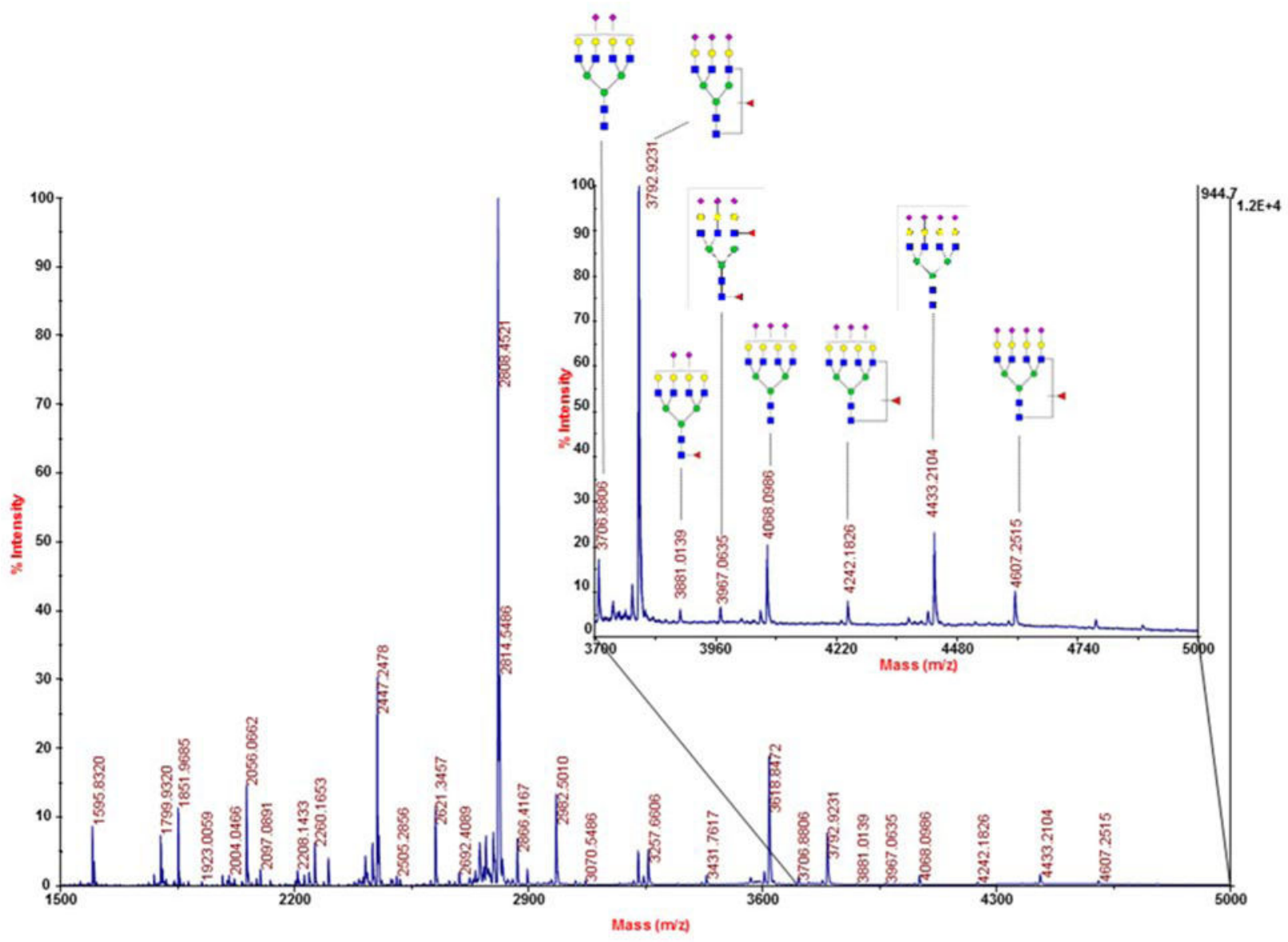

Figure 1.

Representative comprehensive MS glycomic profiles of a colorectal cancer sample. The insets show the high mass region where several of the key glycans that differentiated the two groups are located. Symbols represent $\mathrm{N}$-acetylglucosamine (blue square), mannose (green circle), galactose (yellow circle), fucose (red triangle), and $\mathrm{N}$-acetylneuraminic (sialic) acid (purple diamond). 

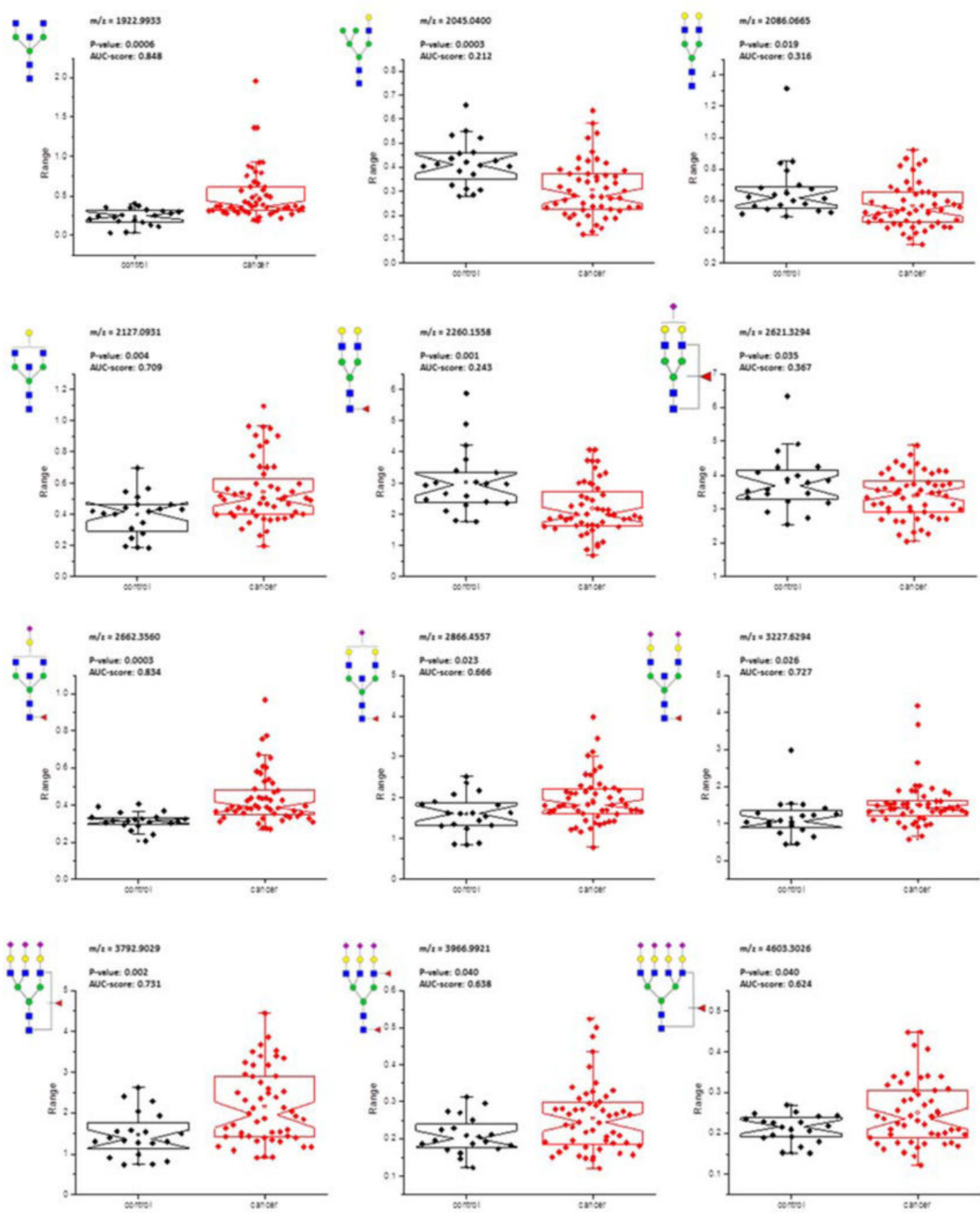

Figure 2.

Notched-box plots depicting changes in the relative abundances of selected glycans passing the different statistical tests in the comprehensive profiles for healthy vs $\mathrm{C} 1$ and $\mathrm{C} 3$ combined. 


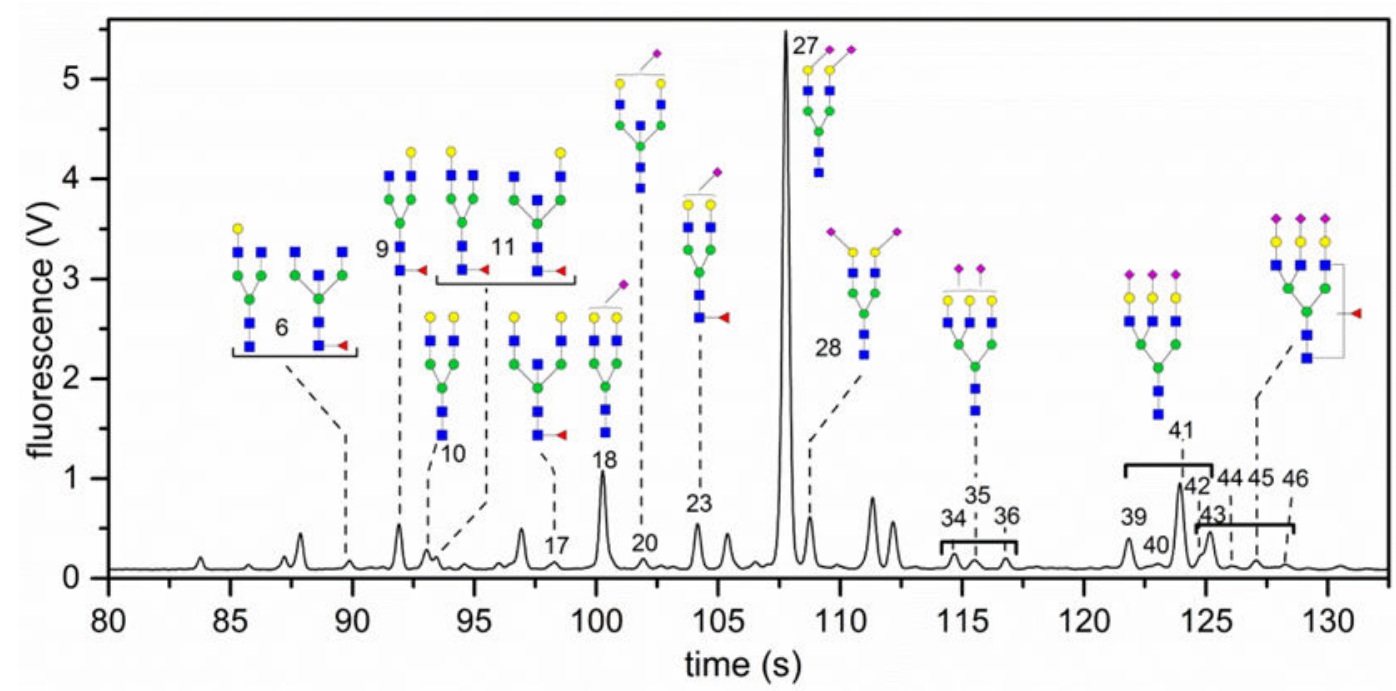

Figure 3.

Annotated microchip electropherogram of serum N-glycans from a colorectal cancer patient. Relevant peaks are numbered and annotated with corresponding structures. 42 peaks were identified in a majority of the samples. Numbers correspond to numbering on a reference electropherogram. 
a)

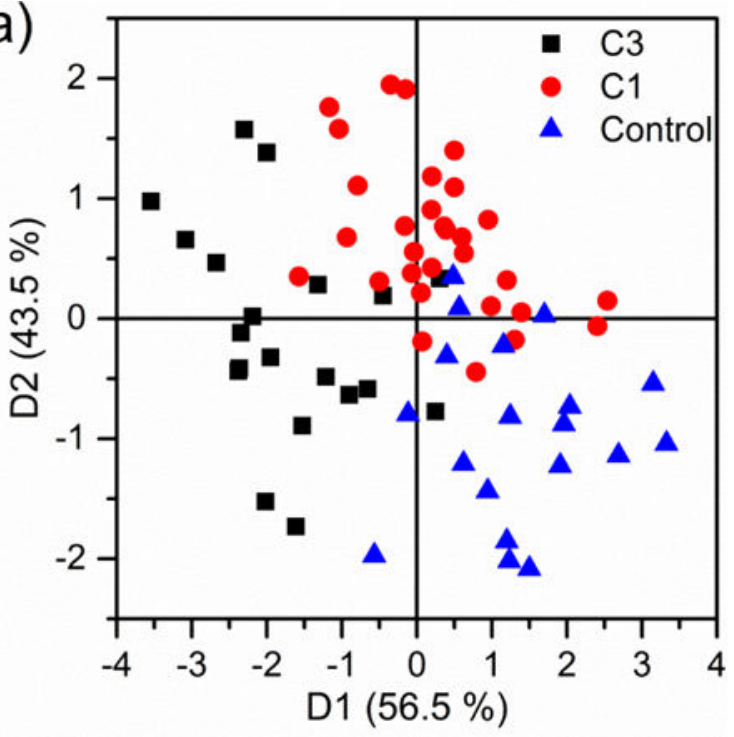

b)

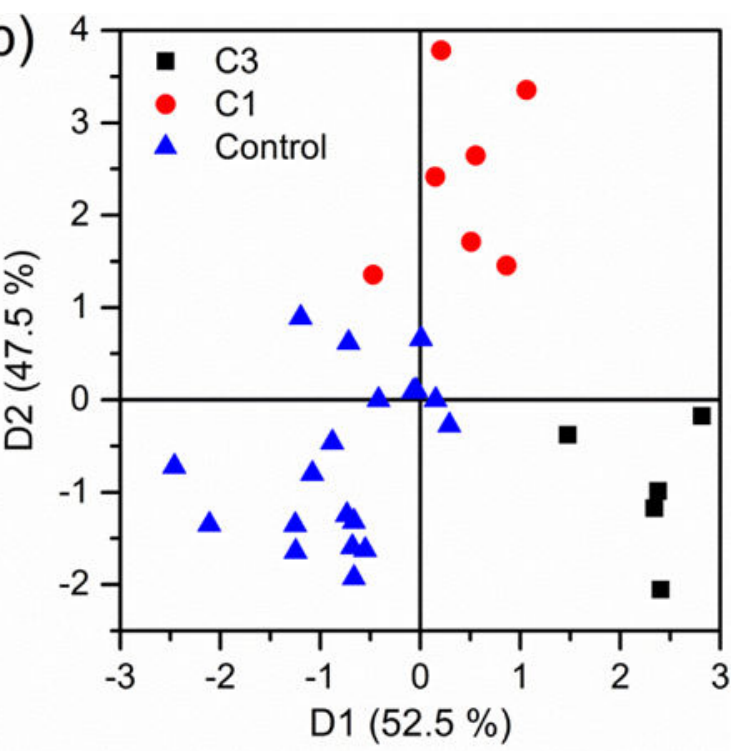

Figure 4.

(a) Principal component analysis (PCA) scores plots for electropherograms of N-glycans derived from colorectal samples from a control group, men and women in the first round of various treatments for colorectal cancer $(\mathrm{C} 1)$, and those same patients after their third round of treatments (C3). The complete sample set shows good differentiation. (b) PCA scores plots for sex (women only) and age-matched (over 60 years old) samples from the $\mathrm{C} 1$ and C3 groups. Greater separation between all sample groups is observed in the sex-and-agematched samples. 


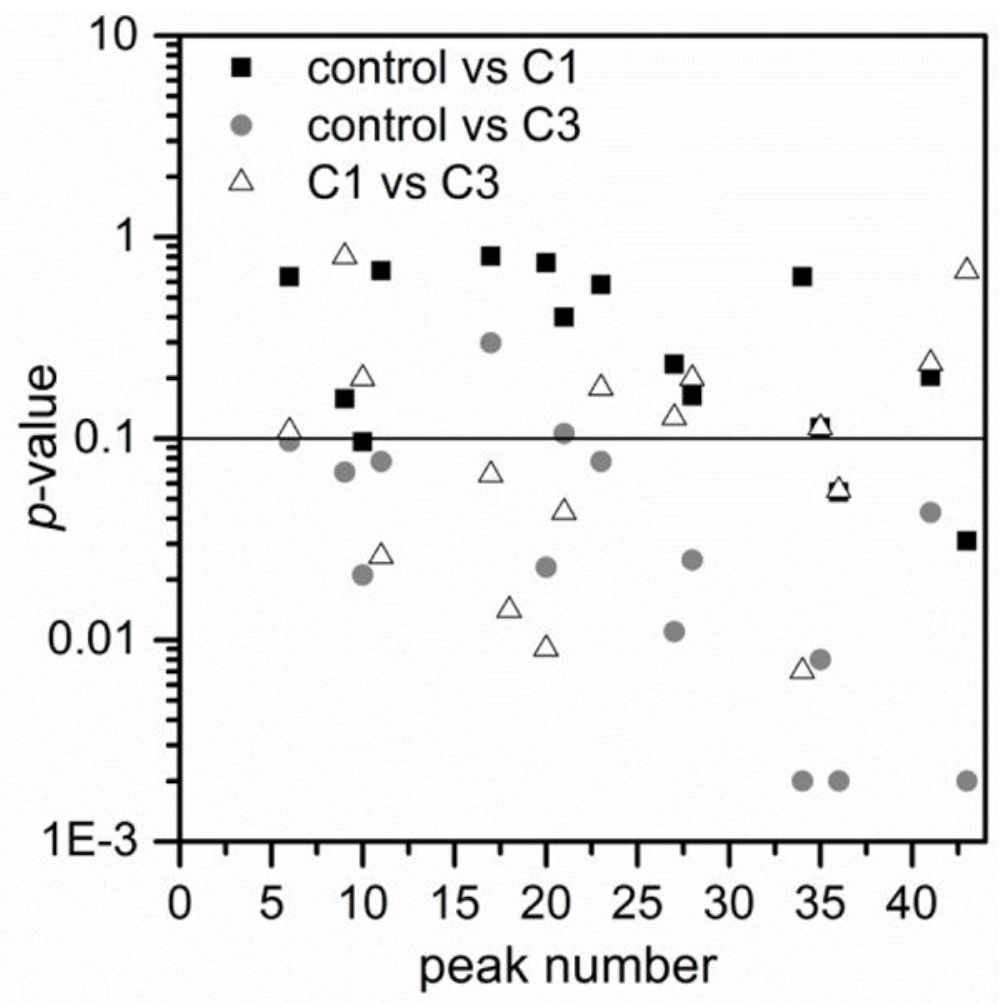

Figure 5.

$p$-Values of $\mathrm{N}$-glycan peaks that showed statistical significance from the analysis of variance (ANOVA) test for pairwise comparisons of the control $(n=20)$ and $C 1$ samples $(n=26)$, control and C3 samples $(\mathrm{n}=16)$, and $\mathrm{C} 1$ and C3 samples. The solid line indicates a $p$-value of 0.1 . For control vs $\mathrm{C} 1$, control vs $\mathrm{C} 3$, and $\mathrm{C} 1$ vs $\mathrm{C} 3,3,14$, and 7 peaks had $p$-values < 0.1 , respectively. 


\section{Table 1}

List of peaks and their associated structures that were statistically significant $(p<0.10)$ for both MALDI-MS and microchip electrophoresis (ME) comparison of control vs cancer (C1 combined with C3) samples. Sixteen different peaks are significant from the comparisons of electrophoresis peak areas by ANOVA analysis for control vs $\mathrm{C} 1, \mathrm{C} 3$, and cancer. Of the significant peaks with identified structures, nine peaks were in agreement with MALDI-MS results.

\begin{tabular}{|c|c|c|c|}
\hline Structure & Peak & ME $p$-value & MALDI $p$-value \\
\hline \multicolumn{4}{|c|}{ Control vs C1 } \\
\hline$=-$ & 10 & 0.096 & 0.078 \\
\hline & 18 & 0.000 & 0.042 \\
\hline 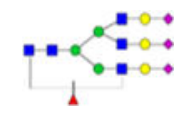 & 43 & 0.031 & 0.078 \\
\hline \multicolumn{4}{|c|}{ Control vs C3 } \\
\hline$=$ & 9 & 0.068 & 0.012 \\
\hline$\because-0$ & 10 & 0.021 & 0.023 \\
\hline 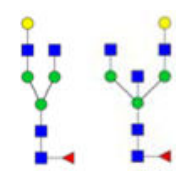 & 11 & 0.077 & $0.012,0.494$ \\
\hline 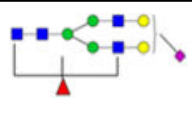 & 23 & 0.077 & 0.0004 \\
\hline$=-\infty$ & 43 & 0.002 & $8.0 \mathrm{E}-06$ \\
\hline \multicolumn{4}{|c|}{ Control vs Cancer } \\
\hline$: 0$ & 8 & 0.072 & $0.027,0.004$ \\
\hline$=-\infty_{0}^{0}=$ & 15 & 0.000 & 0.092 \\
\hline
\end{tabular}

